Poniendo a prueba la teoría de la reproducción del capital cultural en Colombia. El caso de las artes escénicas, los conciertos y el cine

Nora Elena Espinal-Monsalve, Andrey David Ramos-Ramírez y Luz Yadira Gómez-Hernández 
Lecturas de Economía, 92 (enero-junio 2020), pp. 101-131

Nora Elena Espinal-Monsalve, Andrey David Ramos-Ramirez, Luz Yadira Gómez-Hernández

Poniendo a prueba la teoría de la reproducción del capital cultural en Colombia. El caso de las artes escénicas, los conciertos y el cine

Resumen: Tomando como referencia el enfoque teórico de la reproducción social y la transferencia intergeneracional de capital cultural de Bourdieu, en este artículo se evalúa el efecto causal de la participación cultural de los jefes de hogar sobre la participación cultural de los jóvenes en teatro, danza u ópera, conciertos y cine en Colombia. Utilizando los datos de la Encuesta de Consumo Cultural del 2014, se aplican tres metodologias: tratamientos endógenos, propensity score matching e inverse probability weighting. Los resultados aportan evidencia sobre la hipótesis de la transferencia intergeneracional del capital cultural, en la medida en que se observa que los jóvenes que conviven en hogares con jefes de hogar que participan en las actividades analizadas, tienen mayores probabilidades de participar en esas mismas actividades. Concretamente, se encontró que cuando el jefe de hogar asiste a eventos de las artes escénicas, la probabilidad de que los jóvenes en ese hogar también asistan crece entre 34,92\% y 40,90\%; en el caso de los conciertos el efecto es de entre 24,56\% y 25,81\%, mientras que para el cine varía entre $47,57 \%$ y 48,96\%. Estos hallazgos pueden ser un insumo para diseñar y orientar políticas que permitan seguir acortando las brechas de participación cultural en el país. Este es el primer estudio en probar la magnitud de esta teoría y verificarla empiricamente en América Latina.

Palabras clave: economía de la cultura; consumo cultural; capital cultural; evaluación de impacto; efectos de tratamiento.

Clasificación JEL: C21, D04, D12, Z10.

Testing the cultural capital reproduction theory in Colombia. Case of performing arts, concerts and cinema

Abstract: Based on Bourdieu's theory of social reproduction and intergenerational transfer of cultural capital, this paper aims to establish the causal effect of the head of household's cultural participation on the cultural participation of the Colombian youth, specifically in theater, dance or opera, concerts and cinema, using data from the Cultural Consumption Survey 2014 and applying impact evaluation methods such as endogenous treatments, propensity score matching and inverse probability weighting. Results support the intergenerational transfer of cultural capital bypothesis in the sense that youths living in households where the head of household attends cultural activities are more likely to attend the same activities. In fact, we found that when the head of household attends events of performing arts, the probability of attendance of the youngs in the house increases between 34,92\% and 40,90\%; in the case of the concerts the effect is between 24,56\% and 25,85\% whereas for cinema is between $47,57 \%$ and 48,96\%. These findings will allow to continue reducing the cultural participation gaps in Colombia. This is the first study to test the magnitude of this theory and empirically verify it in Latin America.

Keywords: cultural economics; cultural consumption; cultural capital; impact evaluation, treatment effect models.

JEL Classification: C21, D04, D12, Z10.

La mise à l'épreuve de la théorie de la reproduction du capital culturel en Colombie. Le cas du théâtre, des concerts et du cinéma

Résumé: Prenant en compte la théorique de la reproduction sociale et le transfert intergénérationnel du capital culturel proposés par Bourdieu, cet article évalue l'effet causal de la participation culturelle des chefs de famille sur la participation culturelle des jeunes au théatre, aux concerts et au cinéma en Colombie. En utilisant les données de l'Enquête sur la Consommation Culturelle de 2014, trois méthodologies sont appliquées: les traitements endogènes, le propensity score matching et l'inverse probability weighting. Les résultats montrent l'existence d'un transfert intergénérationnel de capital culturel, dans la mesure où les jeunes faisant partis des foyers dont le chef de famille assiste aux activités culturelles, sont plus susceptibles d'assister à ces mêmes activités. Plus précisément, lorsque le chef de famille assiste au théatre, la probabilité que les jeunes de ce foyer assistent également augmente entre 34,92\% et 40,90\%. En ce qui concerne les concerts, l'effet est compris entre 24,56\% et 25,81\%, tandis que pour le cinéma il est compris entre 47,57\% et 48,96\%. Ces résultats peuvent orienter de politiques visant une réduction des écarts concernant l'assistance de la population aux activités culturelles dans le pays. Il s'agit de la première étude en Amérique latine qui teste la théorie reproduction du capital culturel.

Mots clés: économie de la culture; consommation culturelle; capital culturel; évaluation d'impact; effets du traitement

Classification JEL: C21, D04, D12, Z10. 


\title{
Poniendo a prueba la teoría de la reproducción del capital cultural en Colombia. El caso de las artes escénicas, los conciertos y el cine
}

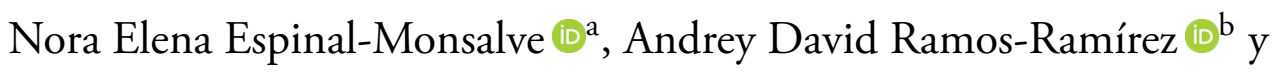 \\ Luz Yadira Gómez-Hernández
}

-Introducción. -I. Metodología. -II. Resultados. -Conclusiones. -Anexo. -Referencias.

doi: 10.17533/udea.le.n92a04

Primera versión recibida el 5 de marzo de 2019; versión final aceptada el 3 de noviembre de 2019

\section{Introducción}

Durante los últimos 30 años, la relación que existe entre la reproducción cultural y el consumo cultural ha sido objeto de estudio desde la sociología, la antropología, los estudios culturales, entre otros, y puede enmarcarse desde la teoría de la reproducción social de Bourdieu (1986) — en la que se centra este artículo - hasta las teorías de la individualización de Bauman (2007) y el omnivorismo cultural de Peterson y Simkus (1992) y Peterson y Kern (1996). Bourdieu (1986) plantea que el capital cultural se transfiere de una generación a otra a través del habitus formado en la familia de origen; este se entiende como las disposiciones o los esquemas de pensar, obrar y sentir asociados a la posición social de los individuos y que pueden explicar la inclinación a determinados estilos de vida en un entorno social homogéneo.

a Nora Elena Espinal-Monsalve: profesora asociada al Departamento de Economía de la Universidad Nacional de Colombia Sede Medellín, Colombia. Dirección electrónica: nespinal@unal.edu.co. https://orcid.org/0000-0003-2777-7410

b Andrey David Ramos-Ramírez: docente ocasional del Departamento de Economía de la Universidad Nacional de Colombia Sede Medellín, Colombia. Dirección electrónica: adramosr@unal.edu.co. https://orcid.org/0000-0002-5795-3190

c Luz Yadira Gómez-Hernández: integrante del grupo de investigación Economía, cultura y políticas de la Universidad Nacional de Colombia Sede Medellín, Colombia. Dirección electrónica: lygomezh@unal.edu.co.https://orcid.org/0000-0002-1791-5886 
Espinal-Monsalve, Ramos-Ramírez y Gómez-Hernández: Poniendo a prueba la teoría...

Desde este punto de vista, la participación cultural de los jóvenes está determinada por los antecedentes familiares a través de una transferencia intergeneracional de capital cultural, porque para apreciar los contenidos simbólicos que estas actividades incorporan se requiere de un habitus formado a partir del contacto temprano con elementos del capital cultural en el hogar (Bourdieu, 1984; 1986).

Bourdieu reconoce tres formas de capital cultural: objetivado, institucionalizado e incorporado. El capital cultural objetivado se refiere a la posesión de bienes culturales como "pinturas, libros, diccionarios, instrumentos musicales, máquinas” (1986, p. 244). El capital cultural institucionalizado se transmite y adquiere en las instituciones educativas a través de la adquisición de nuevo conocimiento y se reconoce con alguna credencial académica. El capital cultural incorporado, el cual consideró como el más importante, es la disposición de la mente y el cuerpo del individuo hacia el consumo cultural; este tipo de capital se adquiere a través de la socialización en la familia siempre que los padres lo posean (Bourdieu, 1986; Willekens \& Lievens, 2014; Notten Lancee, van De Werfhorst \& Ganzeboom, 2015; Georg, 2016).

La concepción del capital cultural incorporado sugiere que un estilo de vida cultural activo de los padres afecta la participación cultural de los niños y adolescentes o, como han planteado algunos investigadores, que el capital cultural se transfiere entre generaciones (van Hek \& Kraaykamp, 2015; Willekens \& Lievens, 2014). Así, las experiencias culturales compartidas con los padres a temprana edad se convierten en un factor determinante en la formación del capital cultural de los futuros adultos, al hacerse parte natural del habitus formado en la familia. Para Bourdieu, el habitus se mantiene constante a lo largo de toda la vida (histéresis) (Georg, 2016), lo que convierte a la transferencia intergeneracional de capital cultural incorporado en un mecanismo eficaz en la reproducción social de las clases dominantes y en la transmisión de desigualdades sociales.

Aunque es la más estudiada, la posición de Bourdieu tiene limitaciones en la argumentación sobre los mecanismos que explican el consumo cultural y que trascienden aquellos relacionados con la transferencia intergeneracional 
de capital cultural (Chan \& Goldthorpe, 2007; Featherstone, 2000; PascalDaloz, 2013; Sassatelli, 2012; Radakovich, 2014). En este sentido, Lahire (2008) se refiere a un "hombre plural" que no solo reproduce patrones de consumo cultural, sino que va produciendo prácticas y discursos de acuerdo a la situación en la que se encuentre. En esto coincide con Bauman (2007), para quien en la modernidad los individuos se independizan de sus orígenes sociales a partir de un proceso creciente de individualización en el que el consumo cultural se relaciona con una expresión de diversidad cultural y patrones culturales cosmopolitas más que con la desigualdad social.

Peterson y Simkus (1992) y Peterson y Kern (1996) muestran que los individuos de la élite se presentan como consumidores "omnívoros", y que por sus facilidades de acceso pueden consumir actividades culturales tradicionalmente consideradas "populares", lo que implica una restricción a la reproducción cultural. Recientemente, Savage (2015) muestra que en la actualidad las diferencias de clase en los estilos de vida culturales son menos visibles, y distingue entre 7 clases $^{1}$ que no pueden ordenarse totalmente de forma jerárquica, como las planteadas por Bourdieu. En Latinoamérica existe una larga tradición de estudios en este campo desde la teoría de la hibridez del consumo cultural: los trabajos de García-Canclini (1999), Martín-Barbero (1999) y Ortiz (2004) plantean que el consumo cultural está marcado por expresiones tradicionales y vanguardistas, lo mismo que elitistas y populares, como expresión de las identidades sociales híbridas en la región.

Para aportar a este debate, este trabajo se propone medir la magnitud de los alcances de la teoría de la reproducción cultural de Bourdieu en Colombia, utilizando metodologías para cuantificar el efecto causal (EC) de la participación cultural de los jefes de hogar sobre la participación cultural de los jóvenes entre 5 y 17 años. Para esto se utilizan microdatos de la Encuesta de Consumo Cultural del año 2014 (ECC2014) aplicada por el Departamento Administrativo Nacional de Estadística (DANE). El EC se estima para el caso de las artes escénicas (teatro, danza u ópera), conciertos y cine, aplicando los

1 1) La élite; 2) la clase media establecida; 3) la clase media técnica; 4) los nuevos trabajadores pudientes; 5) la clase trabajadora tradicional; 6) los trabajadores de servicios emergentes; y 7) el precariado. 
Espinal-Monsalve, Ramos-Ramírez y Gómez-Hernández: Poniendo a prueba la teoría...

métodos de tratamientos endógenos (TE), propensity score matching (PSM) e inverse probability weighting (IPW).

La disponibilidad de datos ha incrementado el interés en el estudio empírico sobre la hipótesis de la transferencia intergeneracional de capital cultural, pero aún son pocos los estudios que se ocupan de testear la teoría de la reproducción cultural. Hoy, la capacidad de prueba de los elementos centrales de la teoría de Bourdieu sigue siendo vaga (Sullivan, 2002), tanto para el estudio de la adquisición de capital cultural incorporado como para la manera en que puede medirse y operacionalizarse. Las investigaciones realizadas desde la economía muestran que, en efecto, el nivel de educación y el ingreso de los individuos tienen una fuerte influencia en el consumo cultural, por lo que es visto como de dominio de las élites (Lévy-Garboua \& Montmarquette, 2003; Gómez \& Espinal, 2016).

Desde otras disciplinas, Nagel (2010) contrastó si la formación de capital cultural responde a una transferencia intergeneracional de hábitos culturales o a un proceso de movilidad social impulsado por la educación. Utilizando datos para un grupo de jóvenes holandeses con edades entre 14 y 24 años, encontró que la participación cultural de los padres y el nivel de educación afectan de forma independiente la participación en teatro, cabaret, conciertos de música clásica, ballet y museos; los antecedentes familiares resultaron ser más importantes que la educación, lo que sustenta la teoría de la transferencia intergeneracional de hábitos culturales. En esta misma línea, van Hek y Kraaykamp (2015) estudiaron en qué medida y a través de qué mecanismos los comportamientos culturales son transferidos entre generaciones; para esto usaron datos para adolescentes holandeses entre 12 y 15 años. Los autores encontraron que los padres influyen en el comportamiento cultural de los hijos, al introducirlos en las actividades culturales, y que existen efectos indirectos del ejemplo cultural que también son significativos, aunque menos pronunciados que la enseñanza directa.

Willekens y Lievens (2014) encontraron un resultado similar en Flandes, Bélgica. Utilizando información de la participación de los adolescentes en arte y patrimonio (museos o exhibiciones de arte, conciertos de música clásica, teatro, danza o actividades de patrimonio) y conciertos de rock y 
pop, mostraron que la transferencia de capital cultural aplica tanto para las actividades de la alta cultura como para actividades de la cultura popular, aunque en el segundo caso la magnitud del efecto es menor. Reeves (2015), por su parte, exploró cómo los padres motivan a sus hijos a tocar instrumentos musicales y cómo esas motivaciones están asociadas con el nivel educativo de los progenitoes. Analizando información de encuestas semiestructuradas en Inglaterra, encontró que la motivación de los padres en promover en sus hijos la práctica cultural está determinada por los lazos familiares y la percepción sobre el talento natural del hijo; este último factor es común entre los padres que tocan instrumentos y que acompañan en esta actividad a sus hijos.

Recientemente Georg (2016) encontró resultados que cuestionan la relevancia de la teoría de la reproducción cultural para el contexto de Alemania. Estudió a través de qué procesos se transmite el capital cultural, qué tan estable es a lo largo de la vida y si las escuelas contribuyen de forma independiente a desarrollarlo, utilizando datos longitudinales que incluyen información de los padres y sus hijos durante un periodo de 33 años. Como medida de capital cultural de los padres utilizó algunos indicadores como la frecuencia de lectura, el número de libros por hogar y la asistencia a cursos culturales. Sus resultados evidenciaron que más allá de lo que llama la herencia social, el capital cultural que un individuo adquiere hasta los 35 años depende de su desempeño educativo y que, desde este momento de la vida, depende de la educación vocacional/profesional.

Estos estudios emplearon diferentes metodologías según los objetivos de la investigación, la naturaleza de las variables de interés y la forma en que fueron organizados los datos. Nagel (2010) estimó modelos lineales jerárquicos teniendo en cuenta que la muestra no fue seleccionada independientemente, sino que se hizo un muestreo en tres niveles: escuelas, clases y estudiantes. Willekens y Lievens (2014), por su parte, estimaron modelos de regresión logística multinivel con el objetivo de controlar los efectos a nivel individual y a nivel de familia. van Hek y Kraaykamp (2015) y Georg (2016) utilizaron modelos de ecuaciones estructurales (SEM, por sus siglas en inglés) para establecer efectos directos e indirectos simultáneos entre los diferentes mecanismos que operan en la transmisión de capital cultural. Reeves (2015) utilizó un enfoque cualitativo sin recurrir a la estimación de 
Espinal-Monsalve, Ramos-Ramírez y Gómez-Hernández: Poniendo a prueba la teoría...

modelos estadísticos. En ninguno de los trabajos revisados se aplicaron los métodos de inferencia causal que se aplican en el presente estudio.

Este artículo representa una contribución a la literatura sobre transferencia intergeneracional de capital cultural en la medida en que aporta elementos de discusión para la comprensión de las dinámicas de consumo cultural en países en desarrollo, pues, a pesar de existir una tradición respecto al análisis de las dinámicas del consumo cultural en América Latina desde la sociología, no se encontró ningún estudio empírico que cuantificase directamente el efecto de la transferencia intergeneracional de capital cultural como determinante del consumo cultural de los jóvenes. Los resultados que se obtienen son útiles para orientar la política pública de la formación de públicos como estrategia para la reducción de las brechas de participación cultural y la consolidación de una demanda por bienes y servicios culturales que en el largo plazo dinamice el desarrollo local a través del fortalecimiento de un sector con industrias culturales sólidas que sean fuente de empleo e ingreso para la población. Para esto, en los siguientes apartados se presentan los datos, las variables y los métodos estadísticos a aplicar; luego se describen los resultados; y finalmente, se presentan las conclusiones.

\section{Metodología}

\section{A. Datos}

Los datos utilizados provienen de la ECC2014 del DANE, la cual recoge información sobre las decisiones de participación cultural de la población colombiana mayor de 5 años residente en las cabeceras municipales de las regiones: Bogotá, Atlántica, Oriental, Central, Pacífica y Orinoquía-Amazonía. La ECC2014 indaga por la participación en presentaciones y espectáculos, espacios culturales (museos y sitios de interés histórico), publicaciones y audiovisuales, prácticas culturales y hábitos de lectura. En total, contiene observaciones de 33.368 individuos, de los cuales 6.131 tienen edades entre los 5 y 17 años y 27.237 tienen 18 años o más.

Este trabajo se enfoca en la asistencia de los niños y adolescentes a eventos de las artes escénicas (teatro, danza u ópera), conciertos y cine, para 
lo cual se utilizan las respuestas de los individuos con edades entre 5 y 17 años que, según la ley colombiana, son menores de edad $(\mathrm{n}=6.131)$. La representatividad de esta muestra se garantiza porque el diseño muestral de la ECC2014 es probabilístico, estratificado, multietápico y de conglomerados; es decir, cada unidad de la población objetivo tiene una probabilidad de selección conocida y mayor a cero, y las unidades de muestreo se clasifican en grupos homogéneos teniendo en cuenta criterios geográficos y socioeconómicos poco correlacionados entre sí.

\section{B. Variables}

En la evaluación del EC de la participación cultural de los jefes de hogar sobre la participación cultural de los jóvenes entre 5 y 17 años en eventos de las artes escénicas, conciertos y cine, se construyen tres variables binarias de resultado $(y)$ que corresponden a la decisión de asistencia de los jóvenes a las tres actividades culturales mencionadas. Las variables de tratamiento $(w)$, que se supone que tienen un EC sobre las variables de resultado, corresponden a la decisión de participación del jefe del hogar al que pertenece el joven en las mismas actividades culturales. Estas variables también son binarias y toman el valor de 1 para los jóvenes cuyo jefe de hogar asiste a la actividad cultural (grupo de tratamiento) y 0 en otro caso (grupo de control).

Debido a la naturaleza binaria de la variable de respuesta, el EC de interés se mide en términos de diferencias en la probabilidad de asisitir a los eventos mencionados entre los jóvenes cuyo jefe de hogar participa en estas actividades culturales y los jóvenes cuyo jefe de hogar no lo hace. Así, el EC para el i-ésimo individuo $\left(\delta_{i}\right)$ se define como:

$$
\delta_{i}=\operatorname{Pr}\left(y_{i}=1 \mid w_{i}=1\right)-\operatorname{Pr}\left(y_{i}=1 \mid w_{i}=0\right) .
$$

Debido a que no es posible observar al mismo individuo en los dos estados de la variable de tratamiento, la estrategia de estimación del EC tiene un problema de observaciones faltantes (Cerulli, 2015). Una posible solución son los diseños experimentales, en los cuales la asignación de la variable de tratamiento es aleatoria, lo que asegura que no existan diferencias sistemáticas entre los grupos de tratamiento y de control antes de la asignación 
Espinal-Monsalve, Ramos-Ramírez y Gómez-Hernández: Poniendo a prueba la teoría...

del tratamiento (Stuart \& Rubin, 2008). Sin embargo, en las ciencias sociales es difícil lograr diseños experimentales debido a que la no aleatoriedad es muy frecuente en la asignación de las variables de tratamiento o porque los programas tienden a estar diseñados para una población determinada y los individuos pueden estar interesados en autoseleccionarse como participantes (Angrist \& Pischke, 2008).

En la literatura empírica se argumenta que la participación cultural es una decisión individual no aleatoria en la que influyen factores como las restricciones de ingreso y tiempo, las características sociodemográficas, el nivel de capital cultural acumulado y el estatus social, entre otros (Borgonovi, 2004; Ateca-Amestoy, 2009; Falk \& Katz-Gerro, 2016; Gómez \& Espinal, 2016; Ramos, 2017), lo que implica que en el presente estudio los individuos autoseleccionan la variable de tratamiento. La no aleatoriedad en la participación cultural genera sesgos de selección debido a que los individuos en los dos grupos pueden tener diferentes características observables (selección en observables) o no observables (selección en no observables) que afectan tanto la variable de tratamiento como la variable de resultado.

El reto que enfrenta el análisis de inferencia causal en este contexto consiste en aplicar métodos que permitan identificar el EC removiendo los posibles sesgos. Los cuasi-experimentos, que permiten crear grupos de beneficiarios y de control con características similares, se constituyen así en una herramienta para estimar el efecto de una variable de tratamiento sobre una de variable resultado en presencia de sesgos de selección (Angrist \& Pischke, 2008; Khandker, Koolwal \& Saman, 2010). Estos métodos permiten estimar el contrafactual, es decir, lo que hubiese sucedido con un individuo de haber recibido el otro estado de la variable de tratamiento, partiendo de un sistema de ecuaciones estructurales conformado por una ecuación para la variable de respuesta y/o una ecuación para la variable de tratamiento de la siguiente manera:

$$
\begin{aligned}
y_{i}^{*} & =\alpha_{1}+\delta w_{i}+x_{i 1}^{T} \beta_{1}+\varepsilon_{i 1} \\
y_{i} & =1\left[y_{i}^{*}>0\right],
\end{aligned}
$$




$$
\begin{aligned}
& w_{i}^{*}=\alpha_{2}+x_{i 2}^{T} \beta_{2}+\varepsilon_{i 2} \\
& w_{i}=1\left[w_{i}^{*}>0\right],
\end{aligned}
$$

donde $y_{i}^{*}$ es una variable latente observada parcialmente a través de la participación cultural de los jóvenes $\left(y_{i}\right)$ y $w_{i}^{*}$ es otra variable latente observada a través de la participación cultural de los jefes de hogar $\left(w_{i}\right) ; x_{1}$ son los factores observables diferentes a la variable de tratamiento que afectan a la variable de respuesta; $x_{2}$ son los factores observables que determinan el mecanismo de asignación de la variable de tratamiento; $\varepsilon_{1}$ y $\varepsilon_{2}$ son los términos de error; y $\alpha_{1}, \alpha_{2}, \beta_{1}, \beta_{2}$ y $\delta$ son los parámetros a estimar.

Las variables que componen los vectores $x_{1}$ y $x_{2}$ se definen a partir del flujo de relaciones que se presenta en la Figura 1 y que fue construido teniendo en cuenta la disponibilidad de información de la ECC2014 y los hallazgos reportados en la literatura empírica sobre determinantes de la participación cultural y la transferencia intergeneracional de capital cultural. Según se observa, la participación cultural de los jefes de hogar está determinada por sus características individuales (Borgonovi, 2004; Gómez \& Espinal, 2016; Ramos, 2017) y su dotación de capital cultural institucionalizado, que en este estudio se mide por el nivel educativo y la participación en prácticas y talleres culturales (van Hek \& Kraaykamp; 2015; Ateca-Amestoy, 2009; Ramos, 2017). La participación cultural de los jóvenes, además de estar determinada por la participación cultural del jefe de hogar en la misma actividad, también está determinada por las características individuales del joven, su propio capital cultural institucionalizado y variables que describen el entorno familiar y que están asociadas a la formación (habitus) en la familia de origen (Nagel, 2010; Willekens \& Lievens, 2014). Estas relaciones justifican la elección de la metodología de este artículo.

\section{Métodos utilizados para establecer el efecto causal (EC) de interés}

La elección del método para estimar el EC de interés depende del tipo de datos disponibles, las condiciones institucionales y normativas del contexto de estudio y la posible existencia de factores inobservables (Angrist \& Pischke, 
Espinal-Monsalve, Ramos-Ramírez y Gómez-Hernández: Poniendo a prueba la teoría...

2008; Parmeter \& Pope, 2013). Como se argumenta en el apartado anterior, se presume que algunas características observables y no observables del jefe de hogar y del entorno en que convive el joven afectan tanto la asignación del tratamiento como la variable de resultado, asunto que se debe controlar para remover los sesgos en la estimación. En el análisis se aplican los métodos de tratamiento endógeno (TE), propensity score matching (PSM) e inverse probability weighting (IPW); el TE asume selección en no observables, mientras que PSM e IPW asumen selección en observables. Se aplican los tres métodos con el objetivo de comparar los resultados y tener mayor certeza sobre el verdadero EC.

Figura 1. Flujo de relaciones entre las variables

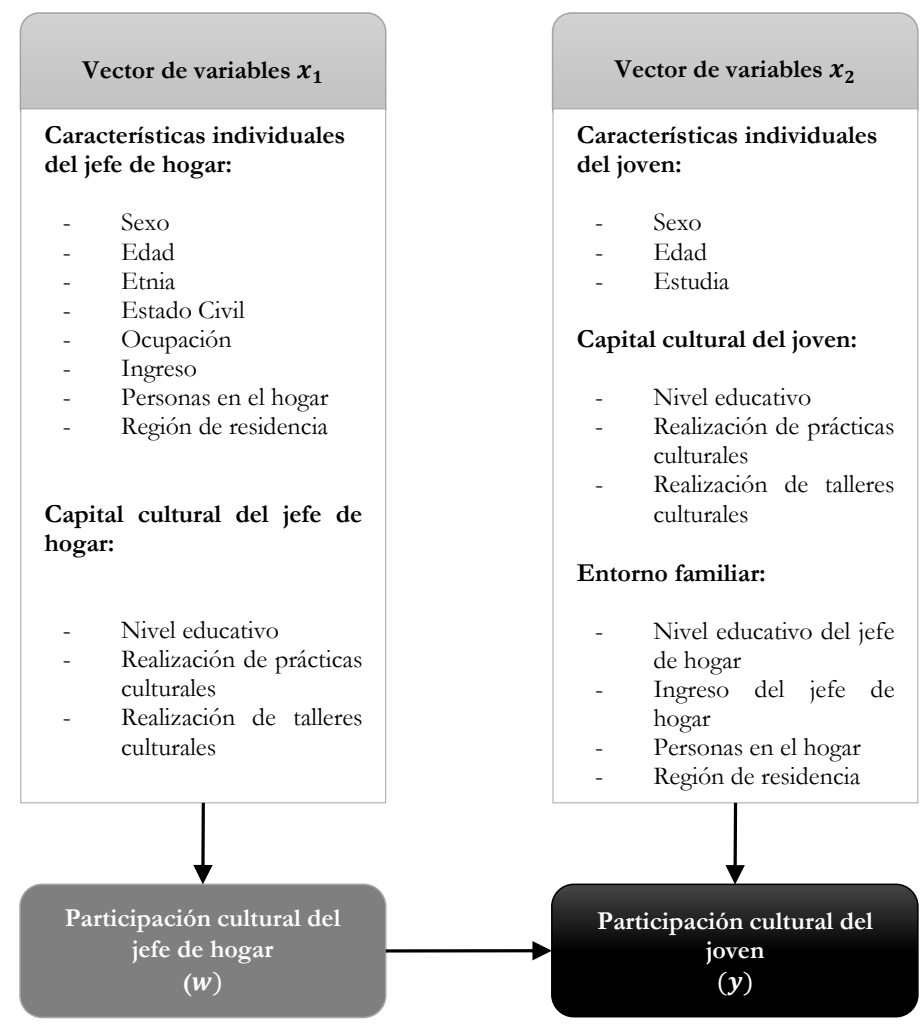

Fuente: elaboración propia. 


\section{Tratamiento Endógeno (TE)}

El método de TE permite estimar el EC de interés cuando existen características no observables que influyen tanto en la asignación del tratamiento como en la variable de resultado. Si este es el caso, se dice que el tratamiento es endógeno y los estimadores de los métodos están basados en selección en observables son sesgados. Para controlar la endogeneidad de la asignación del tratamiento se utiliza un enfoque de función de control en el cual se incluyen los residuales del modelo para la asignación del tratamiento como variables regresoras en el modelo para la variable de respuesta (Wooldridge, 2010; Cerulli, 2015). La aplicación de este método sigue la misma lógica del modelo de selección de Heckman para una variable dependiente binaria, según la cual se modela simultáneamente la ecuación para la asignación del tratamiento y la ecuación para la variable de respuesta y se asume una distribución normal en los componentes de error con coeficiente de correlación $\rho$. Burnett (1997) propuso un procedimiento en dos etapas que facilita los cálculos computacionales:

i) Estimar un modelo probit para la variable de tratamiento y obtener los residuales para cada una de las observaciones.

ii) Estimar un modelo probit para la variable de respuesta agregando como variable explicativa los residuales obtenidos en la primera etapa.

Los cálculos se realizan en el software STATA15 usando el comando eteffects.

\section{Propensity score matching (PSM)}

Los métodos de emparejamiento son herramientas que intentan replicar experimentos aleatorios, pues identifican submuestras de las unidades de control y tratamiento que estén balanceadas con respecto a covariables observadas. El método de PSM es una de estas herramientas, la cual consiste en estimar el contrafactual de cada observación usando el promedio de la variable de respuesta de observaciones similares que reciben el otro nivel de la variable de tratamiento (Stuart \& Rubin, 2008; Khandker et al., 2010). La similitud se determina con base en el propensity score (PS), esto es, la probabilidad estimada de recibir el tratamiento. En este estudio el PS 
Espinal-Monsalve, Ramos-Ramírez y Gómez-Hernández: Poniendo a prueba la teoría...

corresponde a la probabilidad estimada de que el jefe de hogar asista a la actividad cultural. Conforme a lo expuesto por Cerulli (2015), el proceso de aplicación del método es:

i) Estimar la ecuación para la variable de tratamiento a través de un modelo probit y calcular el PS para cada una de las observaciones.

ii) Identificar las observaciones en diferentes estados de la variable de tratamiento con los PS más cercanos y emparejarlas.

iii) Verificar que, una vez se realice el emparejamiento, las variables con que se estima el PS se encuentren balanceadas entre el grupo de tratamiento y el grupo de control.

iv) Si el balance es satisfactorio, calcular el EC de interés.

Los cálculos se realizan en el software STATA15 usando el comando teffects psmatch.

\section{Inverse probability weighting (IPW)}

El IPW asigna niveles de importancia diferentes a las observaciones como estrategia para evitar sesgos en tratamientos no aleatorios; las ponderaciones se asignan de acuerdo con la probabilidad de recibir el tratamiento dados los contrafactuales del caso (Wooldridge, 2007). En la aplicación es necesario definir modelos para la variable de tratamiento y para la variable de respuesta. Debido a la propiedad doble-robusto, es suficiente con que uno de los dos modelos esté bien especificado para que el estimador del EC sea consistente (Cerulli, 2015). El método se aplica en las siguientes tres etapas:

i) Estimar los parámetros del modelo para la variable de tratamiento a través de un modelo probit y calcular las ponderaciones para cada una de las observaciones.

ii) Estimar un modelo probit para la variable de respuesta con las observaciones ponderadas para cada nivel de la variable de tratamiento y obtener el valor predicho de cada observación.

iii) Calcular el EC de interés comparando el promedio del valor de la variable de respuesta para cada nivel de la variable de tratamiento. 
Los cálculos se realizan en el software STATA15 usando el comando teffects ipwra.

\section{Análisis de resultados}

\section{A. Características de los grupos de tratamiento y de control}

El análisis descriptivo de los datos indica que el 26,28\% de los jóvenes en la muestra asisten a eventos de las artes escénicas, el $25,79 \%$ asiste a conciertos y el $37,87 \%$ asiste a cine. En las tres actividades se evidencia un mayor porcentaje de participación en jóvenes que alcanzaron el nivel educativo de primaria o secundaria y que pertenecen a hogares cuyo jefe de hogar es casado o convive en unión libre, trabajador y con ingresos entre 0 y 1 salario mínimo mensual legal vigente (SMMLV). Respecto al capital cultural, en las tres actividades el nivel educativo y la participación en talleres y prácticas culturales de los jefes de hogar es mayor en el grupo de jóvenes participantes. El porcentaje de participación de los jóvenes es superior al de los jefes de hogar en las tres actividades: el 11,24\% de los jefes de hogar asiste a eventos de las artes escénicas, el 24,50\% asiste a conciertos y el 20,89\% asiste a cine (ver Tabla 1). Los jóvenes que pertenecen a hogares donde el jefe de hogar asiste a la actividad conforman el grupo de tratamiento, mientras que el grupo de control lo conforman los jóvenes cuyos jefes de hogar no asisten.

Las diferencias más importantes en las características del jefe de hogar entre los grupos de tratamiento y control se observan en el capital cultural institucionalizado, la etnia, el nivel de ingreso, la ocupación y la región de residencia. En las tres actividades se evidencia que en el grupo de tratamiento es mayor la proporción de jóvenes cuyos jefes de hogar tienen niveles de formación académica superior y de posgrado, que asisten a talleres y prácticas culturales, que tienen ingresos superiores a 1 SMMLV y que son trabajadores. En el caso de la etnia y la región de residencia se observan hallazgos diferentes según la actividad cultural; por ejemplo, se resalta que la proporción de jóvenes en el grupo de tratamiento que residen en Bogotá es significativamente mayor para la asistencia a cine. De otro lado, en la asistencia a conciertos sobresale la 
Espinal-Monsalve, Ramos-Ramírez y Gómez-Hernández: Poniendo a prueba la teoría...

mayor proporción de jóvenes en el grupo de tratamiento cuyos jefes de hogar se reconocen como pertenecientes a una etnia.

Tabla 1. Participación de los jóvenes y jefes de hogar en eventos de las artes escénicas, conciertos y cine

\begin{tabular}{cccccc}
\hline \multirow{2}{*}{ Actividad cultural } & & \multicolumn{2}{c}{ Jóvenes } & \multicolumn{2}{c}{ Jefe de hogar } \\
\cline { 3 - 6 } & & No. & $\%$ & No. & $\%$ \\
\hline \multirow{2}{*}{ Artes escénicas } & No & 4.520 & $73,72 \%$ & 5.442 & $88,76 \%$ \\
& Sí & 1.611 & $26,28 \%$ & 689 & $11,24 \%$ \\
\hline \multirow{2}{*}{ Conciertos } & No & 4.550 & $74,21 \%$ & 4.629 & $75,50 \%$ \\
& Sí & 1.581 & $25,79 \%$ & 1.502 & $24,50 \%$ \\
\hline \multirow{2}{*}{ Cine } & No & 3.809 & $62,13 \%$ & 4.850 & $79,11 \%$ \\
& Sí & 2.322 & $37,87 \%$ & 1.281 & $20,89 \%$ \\
\hline
\end{tabular}

Fuente: elaboración propia con datos del DANE.

\section{B. Resultados}

El desbalance en las características de los grupos de tratamiento y de control señala que la decisión del jefe de hogar de asistir a las actividades culturales no es un hecho aleatorio, sino que está determinado por un proceso voluntario determinado por las características individuales y del capital cultural institucionalizado. Debido a que estas variables también pueden influir en las decisiones de participación de los jóvenes, es necesario aplicar métodos que permitan establecer el efecto causal de interés. A continuación se presentan los resultados de la aplicación de los métodos de TE, PSM e IPW.

\section{Tratamientos endógenos (TE)}

La aplicación del método de TE asume que existen factores no observables dentro del hogar que afectan las decisiones de asistencia a los eventos de las artes escénicas, conciertos y cine, tanto de los jefes de hogar como de los jóvenes. Para verificar este supuesto, se desarrolló 
el procedimiento descrito en el apartado de metodología y se calculó la correlación muestral entre los factores no observables del modelo para la variable de tratamiento y para la variable de respuesta. En la Tabla 2 se presentan los resultados del test de Wald para probar la significancia estadística de las correlaciones estimadas en cada uno de los análisis. En los tres casos la evidencia indica que los factores no observables que intervienen en la asignación del tratamiento no intervienen en la decisión de participación de los jóvenes; es decir, no hay evidencia de selección en los no observables y se prefieren los resultados estimados a través de PSM e IPW que se presentan más adelante (Cerulli, 2015).

Tabla 2. Test de endogeneidad

\begin{tabular}{lcc}
\hline Actividad & Estadístico & Valor $\mathbf{p}$ \\
\hline Eventos de las artes escénicas & 0,18 & 0,9153 \\
Conciertos & 3,69 & 0,1579 \\
Cine & 0,66 & 0,7190 \\
\hline
\end{tabular}

Fuente: elaboración propia con datos del DANE.

\section{Propensity score matching (PSM)}

El PS fue calculado a través de un modelo probit en el que la variable dependiente es la asistencia del jefe de hogar a la actividad cultural, y las variables explicativas corresponden a las características individuales y del capital cultural institucionalizado (ver Figura 1). Los resultados de los modelos se presentan en la Tabla 3. La significancia global indica que las variables explicativas permiten modelar adecuadamente las decisiones de participación cultural de los jefes de hogar. Al analizar la significancia individual de cada una de estas variables se encuentran resultados similares a los reportados en los estudios empíricos aplicados a nivel nacional e internacional (Borgonovi, 2004; Ateca-Amestoy, 2009; Ramos, 2017; entre otros). 
Espinal-Monsalve, Ramos-Ramírez y Gómez-Hernández: Poniendo a prueba la teoría...

Tabla 3. Modelos para estimar el PS

\begin{tabular}{|c|c|c|c|c|c|c|c|}
\hline \multirow{2}{*}{\multicolumn{2}{|c|}{ Características del jefe de hogar }} & \multicolumn{2}{|c|}{ Artes escénicas } & \multicolumn{2}{|c|}{ Conciertos } & \multicolumn{2}{|l|}{ Cine } \\
\hline & & Coeficiente & $\begin{array}{l}\text { Error } \\
\text { estándar }\end{array}$ & Coeficiente & $\begin{array}{c}\text { Error } \\
\text { estándar }\end{array}$ & Coeficiente & $\begin{array}{l}\text { Error } \\
\text { estándar }\end{array}$ \\
\hline $\begin{array}{l}\text { Sexo (Mascu- } \\
\text { lino) }\end{array}$ & Sexo Femenino & $-0,0650492$ & 0,0675036 & 0,0311993 & 0,05294 & $-0,0355984$ & 0,0619933 \\
\hline \multirow{2}{*}{$\begin{array}{l}\text { Edad (12-24 } \\
\text { ańos) }\end{array}$} & 25-65 ańos & 0,0785345 & 0,1551799 & $-0,1620539$ & 0,11002 & $-0,048066$ & 0,128324 \\
\hline & Más de 65 años & $-0,0263533$ & 0,1903178 & $-0,3938775^{* * *}$ & 0,14031 & $-0,5489093^{* * *}$ & 0,1817859 \\
\hline $\begin{array}{l}\text { Etnia (No } \\
\text { pertenece a } \\
\text { unaetnia) }\end{array}$ & $\begin{array}{l}\text { Pertenece a una } \\
\text { etnia }\end{array}$ & $-0,0040209$ & 0,0617404 & $0,0790288^{*}$ & 0,04711 & $-0,3084417^{* * *}$ & 0,0624205 \\
\hline \multirow{4}{*}{$\begin{array}{l}\text { Estado civil } \\
\text { (Soltero) }\end{array}$} & Unión libre & $-0,1460626$ & 0,1017776 & 0,1144767 & 0,08102 & $-0,143927$ & 0,0915197 \\
\hline & Casado & 0,0168431 & 0,1035462 & 0,0481265 & 0,08376 & 0,0024789 & 0,0933743 \\
\hline & $\begin{array}{l}\text { Separado o } \\
\text { divorciado }\end{array}$ & 0,08462 & 0,1014526 & 0,1181162 & 0,08218 & 0,1049123 & 0,0914705 \\
\hline & Viudo & $-0,1496255$ & 0,1488023 & $-0,3598611^{* * *}$ & 0,12082 & $-0,0261979$ & 0,1401464 \\
\hline \multirow{5}{*}{$\begin{array}{l}\text { Ocupación } \\
\text { (Trabajador) }\end{array}$} & Desempleado & 0,0047157 & 0,1542873 & $-0,0524488$ & 0,11325 & 0,0025461 & 0,1442063 \\
\hline & Estudiante & $0,393926^{*}$ & 0,225495 & 0,2044423 & 0,18914 & 0,2386009 & 0,2079051 \\
\hline & Oficios del hogar & 0,0685728 & 0,0807979 & $-0,1958858^{* * *}$ & 0,06274 & 0,0048042 & 0,0760991 \\
\hline & $\begin{array}{l}\text { Incapacitado } \\
\text { permanente }\end{array}$ & $-0,0918907$ & 0,2924034 & $-10,314968^{* * *}$ & 0,44432 & $-0,1894506$ & 0,3079579 \\
\hline & Otra & 0,1025726 & 0,1474684 & $-0,0941884$ & 0,12533 & $-0,3197865^{*}$ & 0,1640954 \\
\hline \multirow{5}{*}{$\begin{array}{l}\text { Ingreso } \\
\text { (Entre 0 } \\
\text { SMMLV y } 1 \\
\text { SMMLV) }\end{array}$} & $\begin{array}{l}\text { Entre } 1 \text { SMMLV y } \\
2 \text { SMMLV }\end{array}$ & $0,30957^{* * *}$ & 0,0571951 & $0,0839995^{* *}$ & 0,04521 & $0,4235196^{* * *}$ & 0,0509582 \\
\hline & $\begin{array}{l}\text { Entre } 2 \text { SMMLV y } \\
3 \text { SMMLV }\end{array}$ & $0,32689^{* * *}$ & 0,0899806 & $0,3372205^{* * *}$ & 0,07690 & $0,5507014^{* * *}$ & 0,0820806 \\
\hline & $\begin{array}{l}\text { Entre } 3 \text { SMMLV y } \\
4 \text { SMMLV }\end{array}$ & $0,43343^{* * *}$ & 0,1226564 & $0,3505521^{* * *}$ & 0,10931 & $0,4171541^{* * *}$ & 0,1126273 \\
\hline & $\begin{array}{l}\text { Entre } 4 \text { SMMLV y } \\
6 \text { SMMLV }\end{array}$ & $0,49879^{* * *}$ & 0,1271452 & $0,3182601^{* * *}$ & 0,11571 & $0,6774417^{* * *}$ & 0,1205317 \\
\hline & Más de 6 SMMLV & $0,57382^{* * *}$ & 0,1588331 & $0,4717177^{* * *}$ & 0,14955 & $1,259193^{* * *}$ & 0,175506 \\
\hline Personas & $\begin{array}{l}\text { Personas en el } \\
\text { hogar }\end{array}$ & $-0,036709^{* *}$ & 0,0145811 & $-0,008784$ & 0,01036 & $-0,0898397^{* * *}$ & 0,0148146 \\
\hline \multirow{5}{*}{$\begin{array}{l}\text { Región de } \\
\text { residencia } \\
\text { (Bogotá) }\end{array}$} & Atlántica & 0,0197449 & 0,0815894 & $0,4591066^{* * *}$ & 0,06607 & $-0,9126721^{* * *}$ & 0,0709751 \\
\hline & Oriental & $0,145896^{*}$ & 0,0810439 & $0,454234^{* * *}$ & 0,06739 & $-0,50557^{* * *}$ & 0,0674356 \\
\hline & Central & $0,32486^{* * *}$ & 0,0825804 & $0,3223895^{* * *}$ & 0,07086 & $-0,507303^{* * *}$ & 0,0705146 \\
\hline & Pacífica & $0,23289^{* * *}$ & 0,0821502 & $0,6591448^{* * *}$ & 0,06754 & $-0,6784412^{* * *}$ & 0,0713146 \\
\hline & Amazonía & $-0,0572861$ & 0,0861014 & $0,2116346^{* * *}$ & 0,06983 & $-1,034707^{* * *}$ & 0,0772202 \\
\hline
\end{tabular}


Tabla 3. Continuación

\begin{tabular}{|c|c|c|c|c|c|c|c|}
\hline \multicolumn{2}{|c|}{ Características del jefe de hogar } & \multicolumn{2}{|c|}{ Artes escénicas } & \multicolumn{2}{|c|}{ Conciertos } & \multicolumn{2}{|c|}{ Cine } \\
\hline & & Coeficiente & $\begin{array}{c}\text { Error } \\
\text { estándar }\end{array}$ & Coeficiente & $\begin{array}{c}\text { Error } \\
\text { estándar }\end{array}$ & Coeficiente & $\begin{array}{c}\text { Error } \\
\text { estándar }\end{array}$ \\
\hline \multirow{4}{*}{$\begin{array}{l}\text { Nivel } \\
\text { educativo } \\
\text { (Primaria) }\end{array}$} & Básica secundaria & $-0,0195676$ & 0,0796203 & 0,0500159 & 0,05632 & $0,5096723^{* * *}$ & 0,0748556 \\
\hline & Media & $0,20775^{* * *}$ & 0,0676776 & $0,1150922^{* *}$ & 0,05071 & $0,8174621^{* * *}$ & 0,0672361 \\
\hline & Superior & $0,55606^{* * *}$ & 0,0731349 & $0,3680885^{* * *}$ & 0,05809 & $1,284572^{* * *}$ & 0,0727768 \\
\hline & Posgrado & $0,92465^{* * *}$ & 0,1219981 & $0,4995624^{* * *}$ & 0,11336 & $1,705908^{* * *}$ & 0,1261057 \\
\hline $\begin{array}{l}\text { Talleres } \\
\text { culturales }\end{array}$ & $\begin{array}{l}\text { Asiste a talleres } \\
\text { culturales }\end{array}$ & 0,1512147 & 0,0960217 & $0,195324^{* *}$ & 0,08645 & $0,2360977^{* *}$ & 0,0969552 \\
\hline \multirow[t]{2}{*}{$\begin{array}{l}\text { Prácticas } \\
\text { culturales }\end{array}$} & $\begin{array}{l}\text { Asiste a prácticas } \\
\text { culturales }\end{array}$ & $0,77841^{* * *}$ & 0,0758609 & $0,3208189^{* * *}$ & 0,07070 & 0,0641055 & 0,0828148 \\
\hline & & \multicolumn{2}{|c|}{$\mathrm{N}=6.131$} & \multicolumn{2}{|c|}{$\mathrm{N}=6.131$} & \multicolumn{2}{|c|}{$\mathrm{N}=6.131$} \\
\hline \multirow{3}{*}{\multicolumn{2}{|c|}{ Medidas de bondad de ajuste }} & \multicolumn{2}{|c|}{ LR Chi2 $=589,86$} & \multicolumn{2}{|c|}{ LR Chi2 $=480,74$} & \multicolumn{2}{|c|}{ LR Chi2 $=1.671,89$} \\
\hline & & \multicolumn{2}{|c|}{ Prob $>$ Chi $2=0,0000$} & \multicolumn{2}{|c|}{ Prob $>$ Chi $2=0,0000$} & \multicolumn{2}{|c|}{ Prob $>$ Chi $2=0,0000$} \\
\hline & & \multicolumn{2}{|c|}{ Pseudo R2 = 0,1369 } & \multicolumn{2}{|c|}{ Pseudo R2 $=0,0704$} & \multicolumn{2}{|c|}{ Pseudo R2 = 0,2660 } \\
\hline
\end{tabular}

Notas: niveles de referencia entre paréntesis. Número de observaciones: 6.131. Significancia: ${ }^{* * *} \mathrm{p}<0,01$; ${ }^{* *} \mathrm{p}<0,05,{ }^{*} \mathrm{p}<0,1$.

Fuente: elaboración propia con datos del DANE.

El capital cultural institucionalizado del jefe de hogar tiene un efecto positivo y significativo sobre la probabilidad de asistencia a los eventos de las artes escénicas, conciertos y cine. En el caso del nivel educativo, por ejemplo, se observa un efecto creciente sobre la probabilidad de asistencia a partir del nivel de formación media, lo cual se evidencia por el incremento en la magnitud de los coeficientes conforme se avanza a niveles superiores de formación. La asistencia del jefe de hogar a talleres y prácticas culturales también resultaron determinantes de la asistencia a conciertos; en cambio, la asistencia a este tipo de talleres no resultó determinante en el modelo para la asistencia a eventos de las artes escénicas ni para el cine.

Respecto a las características individuales, resultan significativos en todos los casos el nivel de ingreso, la región de residencia y el número de personas en el hogar. En comparación con los individuos en el rango más bajo de ingreso (0-1 SMMLV), los jefes de hogar que devengan salarios mayores a 1 SMMLV tienen una probabilidad de asistencia más alta en las tres actividades, hallazgo que refleja el efecto del poder adquisitivo sobre las decisiones de participación 
Espinal-Monsalve, Ramos-Ramírez y Gómez-Hernández: Poniendo a prueba la teoría...

cultural. De manera similar, la probabilidad de asistencia a eventos de las artes escénicas y conciertos es menor para los jefes de hogar que residen fuera de Bogotá, mientras que en el caso del cine esta probabilidad es mayor, hallazgo que puede ser el reflejo de la mayor disponibilidad de salas de cine en la capital del país. De otra parte, incrementos en el número de integrantes en el hogar reduce la probabilidad de asistencia a las tres actividades.

Basados en los modelos anteriores, se estima la probabilidad de que el jefe de hogar asista a cada actividad cultural y posteriormente se emparejan los jóvenes con los jefes de hogar que tengan probabilidades de asistencia similares. El método de emparejamiento utilizado es el de los vecinos más cercanos, para el cual se tuvo en cuenta que se tiene un alto número de individuos en el grupo de jóvenes en hogares cuyo jefe no asiste (grupo de control) y se buscó utilizar toda la información disponible.

En la Tabla 4 se presentan los resultados que muestran que el EC de la asistencia de los jefes de hogar a las tres actividades culturales sobre la probabilidad de asistencia de los jóvenes es positivo y significativo. La magnitud es diferente para cada una de las actividades: cuando el jefe de hogar asiste a cine, la probabilidad de que los integrantes jóvenes en ese hogar también asistan crece en 47,57\%, mientras que para la asistencia a eventos de las artes escénicas y los conciertos el efecto causal estimado es de 40,90\% y $25,81 \%$, respectivamente. La menor magnitud para los conciertos podría explicarse por el hecho de que esta actividad está más expuesta a efectos de socialización y a los medios digitales, por lo cual el mecanismo de transferencia intergeneracional de hábitos culturales sería más débil. No obstante, con los datos disponibles no es posible validar esta hipótesis.

Una vez realizado el emparejamiento se aplicaron pruebas para confirmar que las covariables que determinan la asignación del tratamiento y la variable de resultado están balanceadas en los dos grupos. El análisis de las tablas de balance surgiere que el PSM balancea las características de los dos grupos debido a que, en la mayoría de los casos, las diferencias estandarizadas son cercanas a cero y las razones de varianza se acercan a uno. En variables como nivel educativo, ingreso y número de personas en el hogar, que resultaron ser significativas para explicar la participación del jefe de hogar en las 
actividades culturales y que se presume influyen también en las decisiones del joven, el nivel de balance mejoró significativamente reduciendo las diferencias estandarizadas a menos del $10 \%$. Este análisis es coherente con los gráficos de densidad de la Figura 1A del Anexo, los cuales muestran que cuando se realiza el emparejamiento la distribución de las muestras emparejadas son muy similares para ambos grupos.

Tabla 4. EC promedio estimado con PSM

\begin{tabular}{lcccc}
\hline Actividad & EC Promedio & Error Estándar & Estadístico Z & Valor $\mathbf{p}$ \\
\hline Eventos de las artes escénicas & $0,4090^{* * *}$ & 0,0324 & 12,62 & 0,0000 \\
Conciertos & $0,2581^{* * *}$ & 0,0185 & 13,96 & 0,0000 \\
Cine & $0,4757^{* * *}$ & 0,0623 & 7,64 & 0,0000 \\
\hline
\end{tabular}

Nota: Significancia: ${ }^{* *} \mathrm{p}<0,01 ;{ }^{* *} \mathrm{p}<0,05,{ }^{*} \mathrm{p}<0,1$.

Fuente: elaboración propia con datos del DANE.

El proceso de validación de los resultados del PSM se complementa con la metodología de García-López, Solé-Ollé y Viladecans-Marsal (2015), que consiste en comparar los sesgos estandarizados de la muestra antes y después del emparejamiento. Aunque la muestra inicial parece no tener demasiada contaminación de aspectos irrelevantes porque el sesgo medio en la información inicial no supera el $20 \%$, el emparejamiento mejora los resultados. En la submuestra emparejada el sesgo medio del modelo para Artes escénicas se reduce en cerca del $74 \%$ pasando del 16,7\% al 4,4\%; en el caso de los Conciertos y del Cine la reducción es del $73 \%$ y del $80 \%$, respectivamente (ver Tabla 5).

La aplicación de los métodos basados en selección en observables requiere también del cumplimiento del supuesto de soporte común, el cual plantea que cada individuo tiene una probabilidad positiva de recibir cada nivel de la variable de tratamiento (Khandker et al., 2010; Cerulli, 2015). En los gráficos de barra de la Figura 2 se visualizan las observaciones del grupo de tratamiento y de control que están dentro y fuera del soporte común. En el análisis para los eventos de las artes escénicas se identificaron 213 individuos del grupo de control y 6 del grupo de tratamiento fuera del soporte común, 
Espinal-Monsalve, Ramos-Ramírez y Gómez-Hernández: Poniendo a prueba la teoría...

lo que corresponde al 3,57\% de la muestra. En el caso de los conciertos, 71 observaciones del grupo de control y 9 del grupo de tratamiento, que corresponden al 1,30\% de la muestra, están fuera del soporte común. En el análisis de la asistencia al cine, 909 observaciones del grupo de control y 6 del grupo de tratamiento, que corresponden al 14,92\% de la muestra, están fuera del soporte común. Estas pruebas ratifican que el supuesto se cumple y que las estimaciones del PSM son válidas.

Tabla 5. Reducción del sesgo en la muestra no emparejada y emparejada

\begin{tabular}{lccc}
\hline Actividad & Muestra & Mean Bias & Median Bias \\
\hline \multirow{2}{*}{ Eventos de las artes escénicas } & No emparejada & 16,7 & 14,7 \\
& Emparejada & 4,4 & 3,3 \\
\hline \multirow{2}{*}{ Conciertos } & No emparejada & 11,8 & 10,0 \\
& Emparejada & 3,2 & 2,6 \\
\hline \multirow{2}{*}{ Cine } & No emparejada & 21,5 & 20,9 \\
& Emparejada & 4,3 & 3,4 \\
\hline
\end{tabular}

Fuente: elaboración propia con datos del DANE.

Figura 2. Observaciones fuera y dentro del soporte común

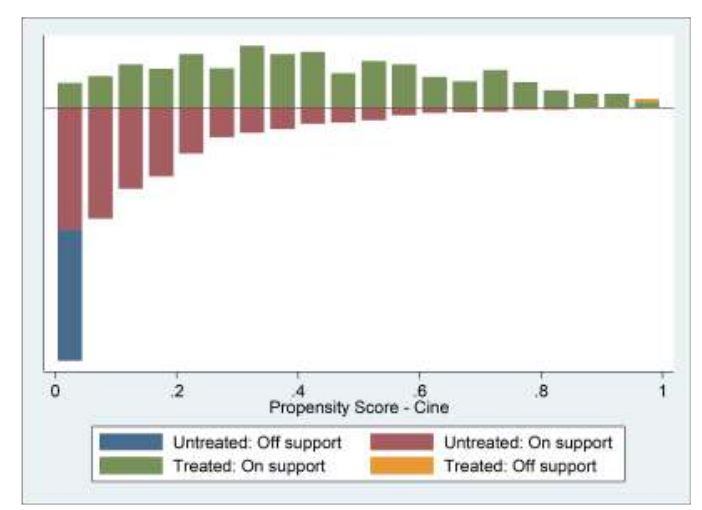


Figura 2. Continuación

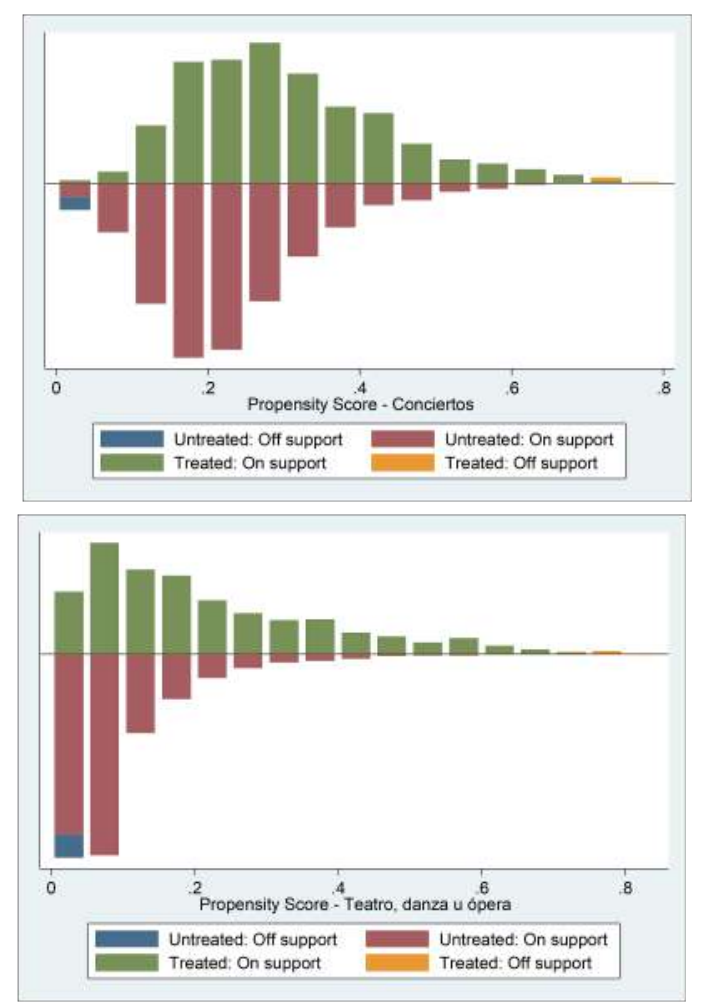

Fuente: elaboración propia con datos del DANE.

\section{Inverse Probability Weighting (IPW)}

Las ponderaciones de las observaciones para la aplicación del método IPW se construyen con base en el PS estimado con los modelos probit que se presentan en la Tabla 3; por tanto, se consideran válidas las mismas pruebas de soporte común de la Figura 2. Una vez ponderadas las observaciones, se estima un modelo probit para la asistencia de los jóvenes a las tres actividades culturales, definiendo las variables explicativas según el flujo de relaciones entre variables de la Figura 1. Los resultados del EC estimados en este caso muestran que la asistencia de los jefes de hogar a eventos de las artes escénicas, conciertos y cine tiene un EC positivo y significativo sobre la probabilidad 
Espinal-Monsalve, Ramos-Ramírez y Gómez-Hernández: Poniendo a prueba la teoría...

de asistencia de los jóvenes a estas mismas actividades. Cuando el jefe de hogar asiste a cine, la probabilidad de que los individuos jóvenes de ese hogar también asistan crece en 48,96\%; esta es la actividad con el EC más grande. En el caso de las actividades de las artes escénicas y de los conciertos, el EC es de 34,92\% y 24,56\%, respectivamente (ver Tabla 6). Estos resultados son similares a los obtenidos con PSM, lo que indica que los métodos de selección en observables son robustos en la identificación del EC de interés.

Tabla 6. EC promedio estimado con IPW

\begin{tabular}{lcccc}
\hline Actividad & EC Promedio & Error estándar & Estadístico Z & Valor $\mathbf{p}$ \\
\hline Eventos de las artes escénicas & $0,3492^{* * *}$ & 0,0260 & 13,42 & 0,0000 \\
Conciertos & $0,2456^{* * *}$ & 0,0146 & 16,80 & 0,0000 \\
Cine & $0,4896^{* * *}$ & 0,0237 & 20,67 & 0,0000 \\
\hline
\end{tabular}

Nota: significancia: ${ }^{* * *} \mathrm{p}<0,01 ;{ }^{* *} \mathrm{p}<0,05,{ }^{*} \mathrm{p}<0,1$.

Fuente: elaboración propia con datos del DANE.

El análisis de las tablas de balance sugiere que el IPW balancea las covariables: en la mayoría de los casos, las diferencias estandarizadas en las variables observables entre los grupos de tratamiento y control son cercanas a cero y las razones de varianza cercanas a uno. El análisis de balance se complementa con la prueba de Imai y Ratkovic (2014), la cual consiste en examinar las condiciones impuestas por el balance en las covariables como condiciones de sobreidentificación. Los resultados (Tabla 7) señalan que a cualquier nivel de significancia que se tome como referencia, el supuesto del balance se satisface.

Tabla 7. Tests de Imai y Ratkovic

\begin{tabular}{lcc}
\hline Actividad & Estadístico Chi2 & Valor $\mathbf{p}$ \\
\hline Eventos de las artes escénicas & 39,1206 & 0,1501 \\
Conciertos & 42,6211 & 0,1228 \\
Cine & 30,324 & 0,5006 \\
\hline
\end{tabular}

Fuente: elaboración propia con datos del DANE. 


\section{Conclusiones}

En el presente artículo se estimó el EC de la participación cultural de los jefes de hogar sobre la participación cultural de los jóvenes entre 5 y 17 años en eventos de las artes escénicas, conciertos y cine. Utilizando los datos de la ECC2014, se aplicaron las metodologías de tratamientos endógenos (TE), propensity score matching (PSM) e inverse probability weighting $(I P W)$, las cuales permitieron calcular el EC de interés removiendo los posibles sesgos de selección en la decisión de participación de los jefes de hogar que podrían afectar las estimaciones. El análisis realizado constituye un aporte a la literatura empírica sobre transferencia intergeneracional de capital cultural porque propone la aplicación de un enfoque metodológico que no se ha usado en este problema de investigación, lo que expande el abanico de alternativas de medición para la obtención de resultados más confiables en un escenario de datos no experimentales. Además, al ser el primer trabajo que aborda directamente el EC en Colombia y en América Latina, genera elementos de discusión para el avance en la comprensión de las dinámicas de consumo cultural en contextos de países en desarrollo.

Los resultados del análisis evidencian la transferencia intergeneracional de capital cultural planteada por Bourdieu (1986) en la teoría de la reproducción social. Una vez realizadas todas las pruebas de validez de los métodos, se encontró que cuando el jefe de hogar asiste a eventos de las artes escénicas, la probabilidad de que los jóvenes en ese hogar también asistan, según la metodología que se considere, crece entre 34,92\% y 40,90\%; en el caso de los conciertos el EC es de entre 24,56 \% y 25,81\%, mientras que para el cine varía entre 47,57\% y 48,96\%. Estos hallazgos sugieren que un estilo de vida cultural activo por parte de los jefes de hogar es un elemento determinante en las decisiones de participación cultural de los jóvenes en Colombia, lo que refleja la existencia de una transmisión intergeneracional de hábitos culturales asociados al capital cultural incorporado en la familia de origen.

De los resultados obtenidos se extrae información valiosa para formular políticas públicas de fomento a la participación cultural en Colombia, orientadas a cerrar las brechas de participación entre los diferentes grupos sociales. Como muestran los datos, una baja proporción de jefes de hogar 
Espinal-Monsalve, Ramos-Ramírez y Gómez-Hernández: Poniendo a prueba la teoría...

asisten a las actividades culturales; esta participación está determinada por el capital cultural (medido por el nivel educativo y la asistencia a prácticas y talleres culturales) y actúa como mecanismo de transferencia de hábitos culturales desde los jefes de hogar hacia los jóvenes, aspecto que fue descrito y testeado empíricamente por Willekens y Lievens (2014) y van Hek y Kraaykamp (2015). Los jóvenes que conviven en hogares con jefes de hogar que no participan en actividades culturales tienen desventajas iniciales en al acceso a este tipo de bienes, por lo que se requiere de intervenciones de política que faciliten a este tipo de población el contacto con los bienes culturales. Así, posibilitar el acceso de los jóvenes a los bienes y servicios culturales, especialmente de los que conviven en hogares donde no hay un babitus cultural consolidado, contribuye a la formación del capital cultural a temprana edad y, por lo tanto, al consumo cultural; esto permitiría disminuir el efecto de la ransferencia intergeneracional de capital cultural y consolidar una demanda cultural robusta en el largo plazo, con efectos potenciales sobre el desarrollo económico local, dada la capacidad del sector cultural para generar empleo, ingreso y crecimiento.

En futuras investigaciones será interesante abordar otros elementos de la transferencia intergeneracional de capital cultural que permitan avanzar en la comprensión de este proceso en el contexto colombiano y que sobrepasan el alcance de este estudio. En específico, se pueden estudiar los mecanismos a través de los cuales los jefes de hogar transfieren sus hábitos culturales a los individuos jóvenes, como lo hacen van Hek y Kraaykamp (2015). Debido a que la ECC2014 no recoge información sobre si el joven asistió acompañado de su jefe de hogar a la actividad cultural o si su decisión correspondió a un comportamiento de imitación, no es posible establecer en qué medida estos dos mecanismos influyen en sus decisiones de participación. Los efectos causales estimados corresponden a efectos agregados que recogen los dos mecanismos.

El papel que juegan las instituciones educativas y los efectos de la socialización entre amigos y conocidos también pueden ser estudiados con información más detallada acerca de las motivaciones de los jóvenes para participar en las actividades culturales. La disponibilidad de este tipo de información podría ayudar a explicar el hecho de que las tasas de participación 
sean más altas para los jóvenes que para los jefes de hogar, y permitiría entender otros mecanismos a través de los cuales los jóvenes que no pertenecen a familias con cierto habitus cultural se interesan por los bienes y servicios culturales y forman su propio acervo de capital cultural a través de un proceso de movilidad social, tal como lo indagó Nagel (2010). Este tipo de análisis reviste especial interés en el caso de los conciertos, actividad en la que se encontró que el EC de interés es significativamente menor con respecto a las demás actividades y en el que el mecanismo de transferencia intergeneracional de hábitos culturales parece no tener tanta fuerza.

\section{Anexo}

Figura 1A. Gráficos de balance (PSM)

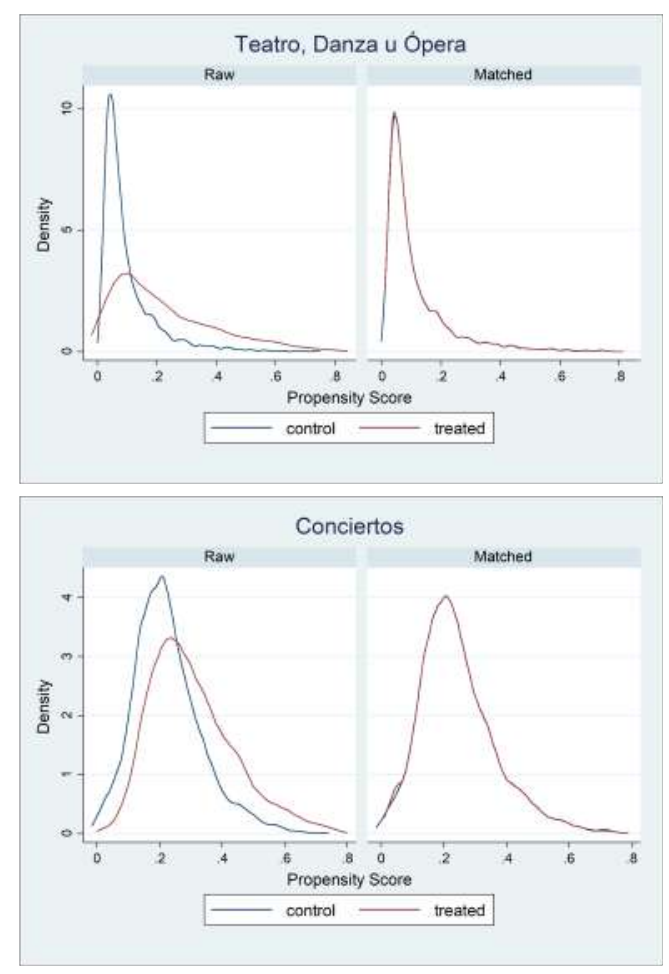


Espinal-Monsalve, Ramos-Ramírez y Gómez-Hernández: Poniendo a prueba la teoría...

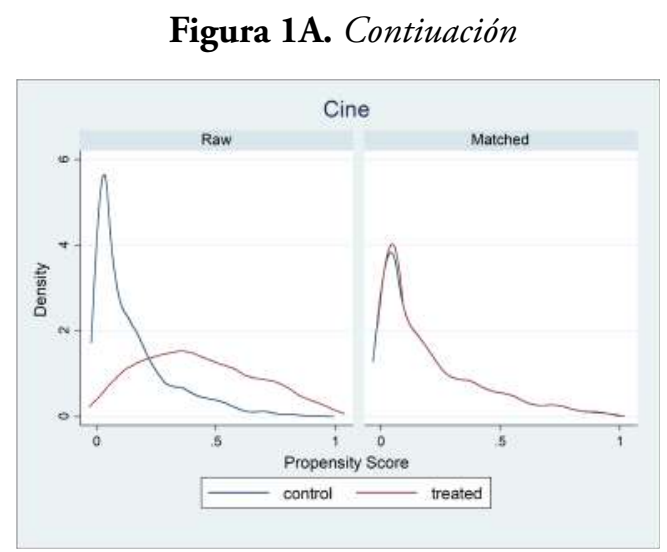

Fuente: elaboración propia con datos del DANE.

\section{Referencias}

Angrist, J. \& Pischke, J.-S. (2008). Mostly harmless econometrics: An empiricist's companion. Princeton, NJ: Princeton University Press.

Ateca-Amestoy, V. (2009). El capital humano como determinante del consumo cultural. Estudios de Economía Aplicada, 27(1), 87-110.

Bauman, Z. (2007). Vida de consumo. Buenos Aires, Argentina: Fondo de Cultura Económica.

Borgonovi, F. (2004). Performing arts attendance: an economic approach. Applied Economics, 36(17), 1871-1885.

Bourdieu, P. (1984). Distinction: A social critique of the judgment of taste. Cambridge, MA: Harvard University Press.

Bourdieu, P. (1986). The forms of Capital. En Richardson, J. (Ed.), Handbook of theory and Research for the Sociology of education. Nueva York: Greenwood.

Burnett, N. (1997). Gender economics courses in liberal arts colleges. Journal of Economic Education, 28(4), 369-376. 
Cerulli, G. (2015). Econometric evaluation of socio-economic programs. Theory and applications. Berlin: Springer.

Chan, T. \& Goldthorpe, J. (2007). Class and Status: the conceptual distinction and its empirical relevance. American Sociological Review, 72(4), 512-532.

Falk, M. \& Katz-Gerro, T. (2016). Cultural participation in Europe: Can we identify common determinants? Journal of Cultural Economics, 40(2), $127-162$.

Featherstone, M. (2000). Cultura de consumo y posmodernismo. BuenosAires, Argentina: Amorrortu.

García-Lopez, M., Solé-Ollé, A. \& Viladecans-Marsal, E. (2015). Does Zoning follow highways? Regional Science and Urban Economics, 53, 145-155.

García-Canclini, N. (1999). Consumo cultural: una propuesta teórica. En: Sunkel, Guillermo (Ed.), El consumo cultural en América Latina (pp. 7295). Santafé de Bogotá, Colombia: Convenio Andrés Bello.

Georg, W. (2016). Transmission of cultural capital and status attainment -an analysis of development between 15 and 45 years of age. Longitudinal and Life Course Studies, 7(2), 106-123.

Gómez, L. \& Espinal, N. (2016). Determinantes de la participación en artes escénicas en Medellín, Colombia. Ensayos de Economía, 26(48), 189207.

Imai, K. \& Ratkovic, M. (2014). Covariate balancing propensity score. Journal of the Royal Statistical Society, Series B, 76(1), 243-263.

Khandker, S., Koolwal, G. \& Saman, H. (2010). Handbook on Impact Evaluation. Quantitative Methods and Practices. Washington DC: The World Bank.

Lahire, B. (2008). The individual and the Mixing of Genres. Cultural Dissonance and Self Distinction. Poetics, 36(2-3), 166-188. 
Espinal-Monsalve, Ramos-Ramírez y Gómez-Hernández: Poniendo a prueba la teoría...

Lévy-Garboua, L. \& Montmarquette, C. (2003). Demand. En: Towse, Ruth (Ed.), A handbook of cultural economics (pp. 201-213). Cheltenham: Edward Elgar Publishing.

Martin-Barbero, J. (1999). Recepción de medios y consumo cultural: travesías. En: Sunkel, Guillermo (Ed.), El consumo cultural en América Latina (pp. 47-71). Santafé de Bogotá, Colombia: Convenio Andrés Bello.

Nagel, I. (2010). Cultural participation between the ages of 14 and 24: intergenerational transmission or cultural mobility? European Sociological Review, 26(5), 541-556.

Notten, N., Lancee, B., van de Werfhorst, H. \& Ganzeboom, H. (2015). Educational stratification in cultural participation: cognitive competence or status motivation? Journal of Cultural Economics, 39(2), 177-203.

Ortiz, R. (2004). Mundialización y cultura. Santafé de Bogotá, Colombia: Convenio Andrés Bello.

Parmeter, C. \& Pope, J. (2013). Quasi-Experiments and Hedonic Property Value Methods. En: List, John y Price, Michael (Eds.), Handbook on Experimental Economics and the Environment (pp. 3-66 ). Cheltenham: Edward Elgar Publishing.

Pascal-Daloz, J. (2013). Rethinking Social Distinction. New York, N.Y.: Palgrave Macmillan.

Peterson, R. \& Simkus, A. (1992). How musical tastes mark occupational status group. En: Lamont y Fournier (Eds.), Cultivating Differences: symbolic boundaries and the making of inequality. Chicago, M.A.: University of Chicago Press.

Peterson, R. \& Kern, R. (1996). Changing highbrow taste: from snob to omnivore. American Sociological Review, 61(5), 900-907.

Radakovich, R. (2014). El gusto revisitado: distinción, hibridez y omnivoridad en el cono sur latinoamericano. Diálogos Possiveis, 13(2), 187-205. 
Ramos, A. (2017). Consumo de bienes del Patrimonio Cultural en Colombia: análisis microeconómico de los determinantes desde los modelos de elección discreta regularizados (Tesis de maestría, Universidad Nacional de Colombia Sede Medellín, Colombia).

Reeves, A. (2015). Music's a family thing: Cultural socialization and parental transference. Cultural Sociology, 9(4), 493-514.

Sassatelli, R. (2012). Consumo, cultura y sociedad. Buenos Aires, Argentina: Amorrortu Editores.

Savage, M. (2015). Introduction to elites From the 'problematic of the proletariat' to a class analysis of 'wealth elites'. The Sociological Review, 63(2), 223-239.

Stuart, E. \& Rubin, D. (2008). Matching with Multiple Control Groups with Adjustment for Group Differences. Journal of Educational and Behavioral Statistics, 33(3), 279-306.

Sullivan, A. (2002). Bourdieu and Education: How Useful is Bourdieu's Theory for Researchers? Netherlands Journal of Social Sciences, 38(2), 144-66.

van Hek, M. \& Kraaykamp, G. (2015). How do parents affect cultural participation of their children?: Testing hypotheses on the importance of parental example and active parental guidance. Poetics, 52, 124-138.

Willekens, M. \& Lievens, J. (2014). Family (and) culture: The effect of cultural capital within the family on the cultural participation of adolescents. Poetics, 42, 98-113.

Wooldridge, J. (2007). Inverse probability weighted estimation for general missing data problems. Journal of Econometrics, 141(2), 1281-1301.

Wooldridge, J. (2010). Econometric Analysis of Cross Section and Panel Data. Cambridge M. A.: MIT Press. 